\title{
Comparison of theoretical methods for the calculation of extended $x$-ray-absorption fine structure
}

\author{
M. Vaarkamp* \\ Laboratory of Inorganic Chemistry and Catalysis, Eindhoven University of Technology, P.O. Box 513, \\ 5600 MB Eindhoven, The Netherlands \\ I. Dring and R. J. Oldman \\ Research and Technology Department, ICI Chemicals and Polymers, P.O. Box 8 The Heath, Runcorn, \\ Cheshire WA7 4QD, United Kingdom \\ E. A. Stern \\ Department of Physics, FM-15, University of Washington, Seattle, Washington 98195 \\ D. C. Koningsberger ${ }^{\dagger}$ \\ Laboratory of Inorganic Chemistry and Catalysis, Eindhoven University of Technology, P.O. Box 513, \\ 5600 MB Eindhoven, The Netherlands
}

(Received 25 February 1993)

\begin{abstract}
Single-scattering calculations of extended x-ray-absorption fine-structure (EXAFS) amplitude and phase were compared with the experimental first-shell data for copper, rhodium, and platinum metal. Theoretical standards used were the tables of Teo and Lee, the tables of McKale et al., and the codes EXCURV90, MUFPOT, and FEFF. The quality of the experimental data was shown to be very high. The experimental first-shell data were obtained by Fourier filtering. The errors introduced in the separation of the first shell from the complete EXAFS spectrum by Fourier filtering were negligible as shown in a model study. The comparison of experimental and theoretical data shows that the accuracy of theoretical standards depends mainly on the treatment of the exchange potential and the energy-dependent losses. The most accurate description of the exchange potential is the energy-dependent HedinLundqvist potential with an energy-dependent self-energy, as used in FEFF. The use of ground state $X \alpha$ or energy-independent exchange, as in the tables of McKale et al. or the codes EXCURV90 and MUFPOT, is found to be inadequate and leads to large phase and amplitude errors. Addition of an energydependent mean free path to the tables of McKale et al. improved the accuracy by $15-25 \%$. The physical reasons for the differences in accuracy of the theoretical methods examined are discussed.
\end{abstract}

\section{INTRODUCTION}

Analysis of extended $x$-ray-absorption fine-structure (EXAFS) spectra requires a knowledge of backscattering amplitudes and phase shifts. They can be extracted by Fourier filtering from the EXAFS spectrum of a compound with known structure or calculated from first principles. Both methods can introduce (systematic) errors in the analysis of unknown compounds. Extraction of backscattering amplitudes and phase shifts from reference compounds suffers from Fourier filtering truncation errors. The accuracy of calculated backscattering amplitudes and phase shifts is hampered by the approximations necessary to make calculations possible. A number of first-principle methods to calculate backscattering amplitudes and phase shifts have been developed. ${ }^{1-5}$ The most widely used computer programs are EXCURV and FEFF. Tables of phase shifts and backscattering amplitudes as compiled by Teo and Lee and McKale et al. are also widely used.

One of the goals of the International Workshop on Standards and Criteria in X-Ray Absorption Spectrosco$\mathrm{py}^{6}$ is to assess quantitatively the applicability of current theoretical models in $\mathrm{x}$-ray-absorption fine-structure (XAFS) analysis. This may be carried out by comparing the available theoretical standards with high-quality experimental spectra from well-characterized materials. Each of these theoretical standards is based on different prescriptions for scattering potentials and self-energies or exchange potentials. By comparing theoretically calculated XAFS spectra with experiment, the relative importance of the key elements embodied in each of the theoretical approaches can be assessed. The most straightforward way of comparing calculated and experimental XAFS is by looking at a single absorberbackscatterer pair involved in a single-scattering process. To isolate the contribution of a single pair to the experimental data, Fourier filtering is necessary.

The aim of this paper is to determine which elements in the various theoretical approaches are most important for accuracy. The calculated phase shift and backscattering amplitude were compared with first-shell experimental spectra. We used only monoatomic metals to avoid the difficulties of charged atoms in calculations. For this purpose a readily available material from each of the three rows of transition metals was selected $(\mathrm{Cu}, \mathrm{Rh}$, and 
Pt foil, respectively).

Accurate experimental data are essential in a comparison of experimental data with theoretical calculations. The quality of the experimental data used was verified by comparison of measurements under different experimental conditions. Separation of the single-scattering firstshell contribution from the complete EXAFS spectrum was carried out by Fourier filtering. Fourier filtering suffers from truncation errors due to the limited data range used in both the forward and the inverse Fourier transform. To verify that Fourier filtering introduces errors which are negligible compared to the differences in the theoretical standards, we carried out a model study to quantify the Fourier filtering errors.

From the statistical errors in the data and the differences between experimental data and theoretical fit, goodness of fit values and errors in the determined parameters are calculated. The goodness of fit values and the number of free parameters are used to test whether the differences in fit quality between the different theoretical references are statistically significant.

\section{DESCRIPTION OF THEORETICAL METHODS}

$\mathrm{X}$-ray absorption fine structure, i.e., the oscillatory structure in the $x$-ray-absorption coefficient, contains quantitative information about the local environment of an absorbing atom. This information includes nearneighbor distances $R$, coordination numbers $N_{R}$, and a pair distribution function about the average which, in simple cases, can be described by a mean-squared deviation $\sigma^{2}$. These quantities appear as structural parameters in the curved-wave XAFS equation ${ }^{7}$ together with other quantities which depend on the electronic structure of the material,

$$
\begin{gathered}
\chi_{l}=-\sum_{R} N_{R} S_{0}^{2} \frac{\left|f_{\mathrm{eff}}(\pi, k, R)\right|}{k R^{2}} \sin \left(2 k R+2 \delta^{c}+\Phi_{\mathrm{eff}}\right) \\
\times e^{-2 \sigma^{2} k^{2}} e^{-2 R / \lambda(k)} .
\end{gathered}
$$

Here $f_{\text {eff }}(\pi, k, R)=\left|f_{\text {eff }}(\pi, k, R)\right| e^{i \Phi_{\text {eff }}}$ is the effective curved-wave backscattering amplitude, ${ }^{7} \delta^{c}$ is the finalstate $l$-wave central atom phase shift, $\lambda(k)$ is the mean free path of the photoelectron, and $S_{0}^{2}$ is a many-body amplitude reduction factor. The determination of structural parameters from experimental data, which is the goal of XAFS analysis, always requires a comparison with an accurately known experimental or theoretical reference system.

The theoretical standards considered in this study include tables of plane-wave XAFS phases and amplitudes of Teo and Lee, ${ }^{1}$ tables of curved-wave XAFS phases and amplitudes of McKale et al., ,,8 and three ab initio computer codes EXCURV90, ${ }^{3}$ MUFPOT, ${ }^{4}$ and FEFF. ${ }^{5}$ Although curved-wave effects are known to be important, we have included tables based on plane-wave amplitudes and phases because they are still widely used.

The tables of McKale et al.,${ }^{8}$ MUFPOT, ${ }^{4}$ and EXCURV90 (Ref. 3) include curved-wave effects exactly, but are based on ground-state exchange-correlation potentials and thus ignore the energy-dependence of the electron. All three require the use of the energy reference or "inner potential" $E_{0}$ as a fitting parameter. The central atom tables of Teo and Lee ${ }^{1}$ are based on a complex Hedin-Lundqvist self-energy and atomic potentials. The ab initio singlescattering code FEFF makes use of an overlapped atom scattering potential and a Hedin-Lundqvist self-energy. Implicit in the use of tables is a reliance on the hypothesis of chemical transferability in which system-dependent chemical effects are neglected. This hypothesis is not well tested by monoatomic metals and requires testing in heterogeneous molecules.

\section{EXPERIMENT}

\section{A. Data collection}

Two sets of data were collected for each foil. The samples were cooled with liquid nitrogen to approximately $-130^{\circ} \mathrm{C}$. The thickness of the foils was 7,4 , and $20 \mu \mathrm{m}$ for copper, platinum, and rhodium, respectively. The measurements were carried out in the transmission mode using ion chambers filled with argon to give a $\mu x$ of $20 \%$ in the first ion chamber and a $\mu x$ of $80 \%$ in the second ion chamber. To reduce noise several scans were averaged for each set of data.

Copper foil data were collected at the Daresbury Synchrotron Radiation Source (SRS) using station 9.2 and 8.1, equipped with flat $\mathrm{Si}$ (220) double crystal monochromators. Station 8.1 was also equipped with vertical and horizontal focusing mirrors. The storage ring was operated with an electron energy of $2 \mathrm{GeV}$ and a current between 120 and $250 \mathrm{~mA}$. The estimated resolution is 2 $\mathrm{eV}$ at the $\mathrm{Cu} K$ edge. The monochromator was detuned to $50 \%$ intensity on station 9.2 and $70 \%$ intensity on station 8.1 to avoid the effects of higher harmonics present in the $x$-ray beam.

Platinum foil data were collected at the SRS, station 9.2, using a flat $\mathrm{Si}(220)$ double crystal monochromator. The estimated resolution was $3 \mathrm{eV}$ at the Pt $L_{\mathrm{III}}$ edge. The monochromator was detuned to $50 \%$ intensity to avoid the effects of higher harmonics present in the x-ray beam. Additional platinum foil data were collected at the Stanford Synchrotron Radiation Laboratory (SSRL), station 1.5, using a $\mathrm{Si}(220)$ channel cut monochromator (3 $\mathrm{GeV}, 40-80 \mathrm{~mA}$ ). To decrease detection of higher harmonics the gas filling of the second ion chamber was decreased. ${ }^{9}$

Rhodium foil data were collected at the SSRL station 1.5, using a Si (220) channel cut monochromator. The estimated resolution was $5 \mathrm{eV}$ at the $\mathrm{Rh} K$ edge. Additional rhodium foil data were collected at the SRS station 9.2, using a Si (220) channel cut monochromator. No precautions were taken to prevent the detection of higher harmonics.

\section{B. Data reduction}

To extract the EXAFS from the x-ray absorption spectrum, the preedge background was approximated by 


$$
\mu x_{\text {preedge }}=\frac{C_{2}}{E^{2}}+\frac{C_{1}}{E}+C_{0}
$$

The edge energy was set to $8980 \mathrm{eV}$ for copper foil at the peak of the $\mathrm{Cu} K$ edge feature. The platinum and rhodium edge energies were set to the maximum in the first derivative in the edge region (11564 and $23220 \mathrm{eV}$ ). The background in the EXAFS region was approximated with a smoothing spline and optimized according to the criteria described by Cook and Sayers. ${ }^{10}$ The spectra were normalized to the edge step at $50 \mathrm{eV}$ behind the edge.

Averaging $\chi$ functions and Fourier filtered $\chi$ functions gives the opportunity to average out statistical errors in background subtraction and Fourier filtering procedures. The errors in both background subtracted $\chi$ functions and Fourier filtered $\chi$ functions were calculated from averages of five, three, and four data sets for copper, rhodium, and platinum foil, respectively.

\section{Calculations}

Parameters used to calculate the first-shell EXAFS spectra are listed in Table I. Distances are taken from Xray diffraction (XRD) measurements. ${ }^{11}$ All compounds have a fcc structure; thus the first-shell coordination number for all compounds is 12 . The Debye-Waller factors for copper and platinum foil were taken from Stern, Bunker, and Heald. ${ }^{12}$ The Debye-Waller factor for rhodium foil has been calculated from the value determined by XRD (Ref. 13) and the displacement correlation function. ${ }^{14}$ The imaginary potentials (VPI) used to describe inelastic losses in EXCURV90 and MUFPOT (Ref. 15) were selected by optimizing fits on foils measured separately at room temperature. Phase shifts and backscattering amplitudes from the tables of Teo and Lee were derived from calculations using Clementi-Roetti wave functions for copper and from the calculations using HermanSkillman wave functions for rhodium and platinum. The average of the ruthenium and palladium results was used for rhodium. Data from the tables of McKale et al. were interpolated on a $1 / R$ grid.

The many-body reduction factor $S_{0}^{2}$ is used as a scaling factor by all theoretical standards. However, the values used differ and are mostly obtained from fitting a compound with known structure with calculated phase shift and backscattering amplitude. We have chosen to set $S_{0}^{2}$ to 1 in all calculations in order to be able to directly relate the coordination numbers obtained in the analysis to the magnitude of the calculated backscattering amplitudes.

TABLE I. Input parameters for theoretical calculations.

\begin{tabular}{cccccc}
\hline Absorber & Backscatterer & $r(\AA)$ & $N$ & $\sigma^{2}\left(\AA^{2}\right)$ & VPI $(\mathrm{eV})$ \\
\hline $\mathbf{P t}^{0}$ & $\mathbf{P t}^{0}$ & 2.774 & 12 & 0.00261 & -8.5 \\
$\mathbf{R h}^{0}$ & $\mathbf{R h}^{0}$ & 2.687 & 12 & 0.00140 & -8.5 \\
$\mathrm{Cu}^{0}$ & $\mathrm{Cu}^{0}$ & 2.560 & 12 & 0.00328 & -5 \\
\hline \hline
\end{tabular}

\section{Method of comparison}

The coordination parameters of the experimental firstshell data were determined using the phase shift and backscattering amplitude calculated with the different theoretical methods. The difference between the experimental first-shell data and the model EXAFS function is described by the $k^{n}$ variance

$$
k_{\text {variance }}^{n}=100 \frac{\int\left\{k^{n}\left[\chi_{\text {model }}(k)-\chi_{\text {expt }}(k)\right]^{2}\right\}}{\int\left\{k^{n}\left[\chi_{\text {expt }}(k)\right]^{2}\right\}} .
$$

An $F$ test on the $\varepsilon_{v}^{2}$ (Ref. 6) was used to test the statistical significance of the differences in $k^{n}$ variance of the model EXAFS functions.

\section{RESULTS AND DISCUSSION}

To compare theoretical and experimental EXAFS spectra, very accurate experimental data are necessary. We identified three possible sources of errors: (i) statistical uncertainty, (ii) Fourier filtering errors, and (iii) errors due to the specific experimental conditions used. The statistical uncertainty (error) in the experimental data used in this study is calculated for the average of a number of data sets. The Fourier filtering errors were estimated by a model study. The errors due to changes in experimental conditions are estimated by comparison of EXAFS spectra measured under different experimental conditions.

\section{A. Raw data and statistical errors}

The $k^{1}$ weighted EXAFS spectra of copper, rhodium, and platinum foil are presented in Fig. 1 together with their $k^{3}$ weighted Fourier transforms. Up to six shells can be distinguished in the Fourier transform implying these are very high-quality EXAFS spectra.

Four scans of platinum foil taken at SRS 9.2 were background subtracted and normalized. Their $\chi$ functions were averaged and the standard deviation $(\sigma$, error $)$ for the average $\chi$ function was calculated. Subsequently all four $\chi$ functions were Fourier filtered to separate the first-shell EXAFS from the other contributions. The resulting Fourier filtered $\chi$ functions were averaged and the standard deviation for the average Fourier filtered $\chi$ function was calculated. The absolute errors in the unfiltered and filtered platinum foil $\chi$ functions are shown in Fig. 2(a). The peaks correspond to nodes in $\chi$. There is a clear decrease in the magnitude of the errors of both raw and Fourier filtered data with increasing $k$ value. The magnitude of the noise in the Fourier filtered data is slightly less than the magnitude of the noise in the raw data. The fact that the noise varies so strongly with $k$ indicates that it is not purely statistical but includes a large fraction of systematic noise. It is likely that the increased magnitude of the errors at low $k$ is caused by the uncertainties in the background subtraction procedure. The relative errors are shown in Fig. 2(b). It is striking that the relative errors in the raw and the Fourier filtered EXAFS spectrum are of the same magnitude over the com- 
plete $k$ range examined. The larger errors in the raw data are compensated by the larger amplitude of the raw EXAFS. The absence of any $k$ dependence in the relative errors is also related to the decrease in absolute error accompanied by a comparable decrease in amplitude of the EXAFS. The average relative error between 2.3 and 19.1 $\AA^{-1}$ is $6 \%$.

\section{B. Fourier filtering truncation errors}

The aim of Fourier filtering is to separate the contribution of a single absorber-backscatterer pair from the contributions of other absorber-backscatterer pairs. This is either to facilitate analysis or to extract experimental phase shifts and backscattering amplitudes from the EXAFS spectrum of a compound with known structure.

To quantify the errors made in this procedure a trial first-shell copper foil EXAFS spectrum was calculated using the phase shift and backscattering amplitude obtained with FEFF. This trial EXAFS spectrum was
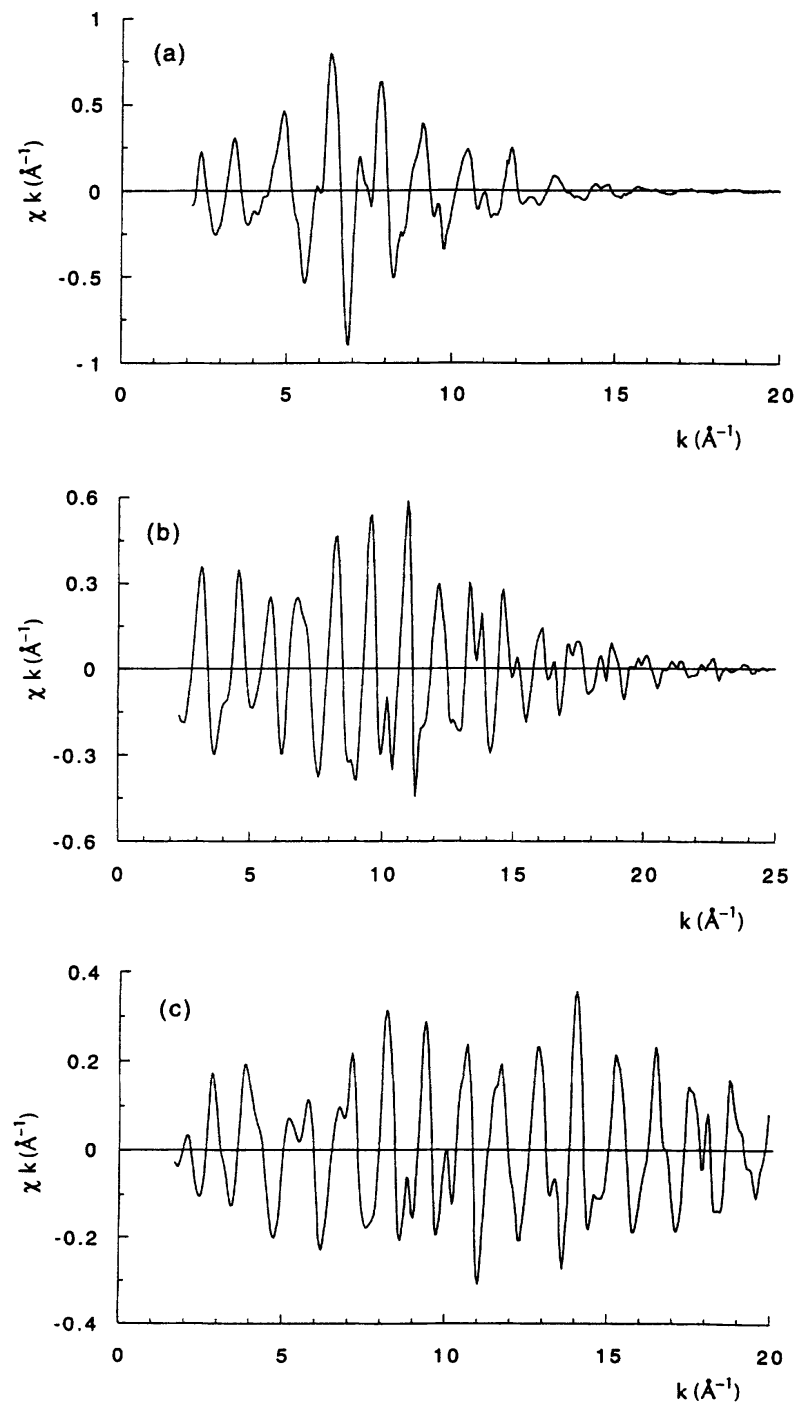

Fourier filtered over a number of forward and inverse ranges. By analyzing the Fourier filtered EXAFS spectrum with the phase shift and backscattering amplitude used to calculate the trial first-shell EXAFS spectrum, filtering errors were quantified.

As Fourier truncation errors are largest at the start and end of the Fourier transform interval, the range and weighting of the analysis were varied. To see the effect of window functions, forward transforms ending and starting at nodes or maxima and minima were compared.

Analysis of a Fourier filtered $\chi$ function obtained with a forward range of $2.3-19.4 \AA^{-1}$ and an inverse of $0-8 \AA$ resulted in a variance of zero, implying that in this case there are no Fourier filtering errors. Analysis of a Fourier filtered $\chi$ function obtained with a forward range of 2.3-19.4 $\AA^{-1}$ and an inverse range of $1.3-3.1 \AA$ resulted in a nonzero variance. Figure 3(a) shows the decrease of the variance upon shortening the interval for analysis. The maximum variance has been calculated over the $k$-space interval $2.4-19.3 \AA^{-1}$. The start and
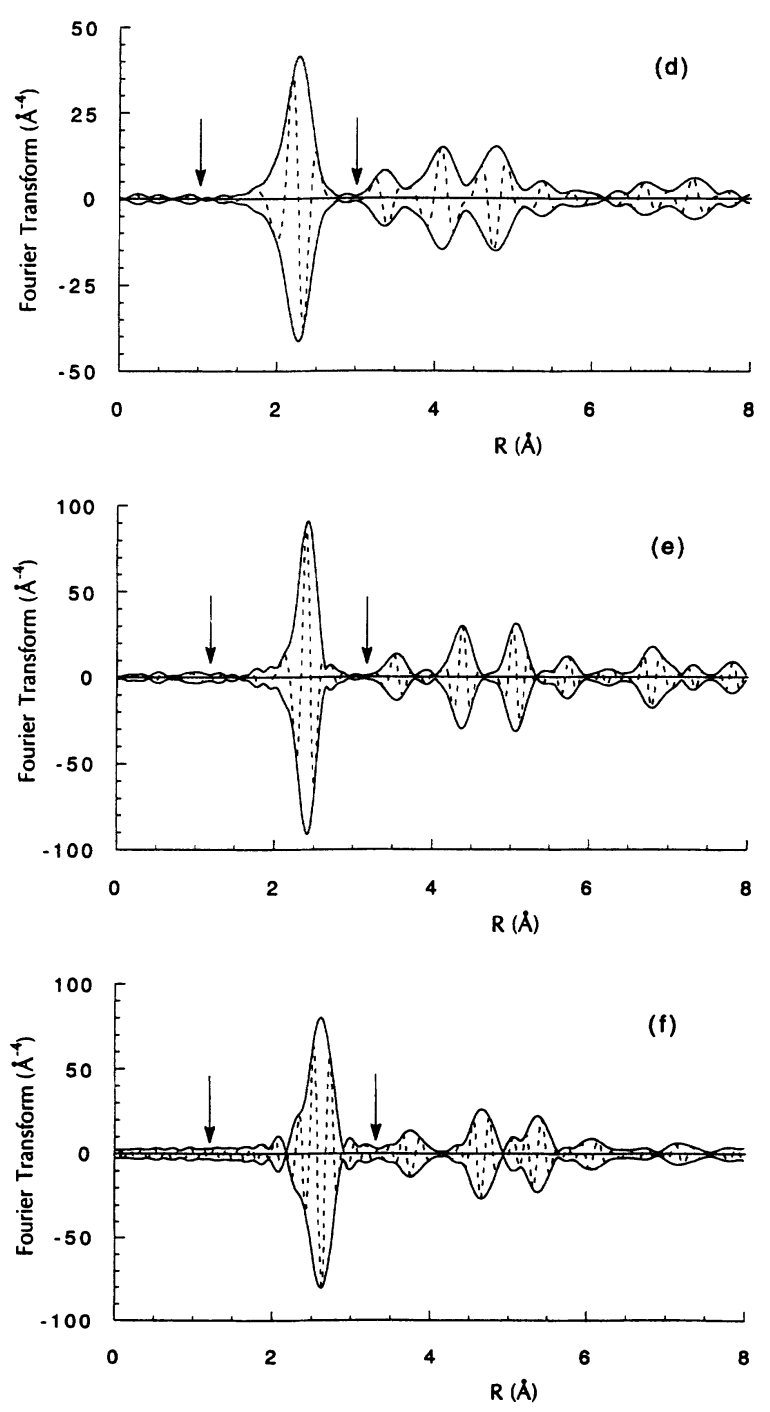

FIG. 1. $k^{1}$ weighted EXAFS spectra of (a) copper foil (SRS 8.1), (b) rhodium foil (SSRL 1.5), (c) platinum foil (SRS 9.2), and their $k^{3}$ weighted Fourier transforms [(d), (e), and (f), respectively]. Fourier transform ranges are listed in Table III. 
TABLE II. Numerical results of the $k^{1}$ weighted analysis of experimental data with a reference measured under different experimental conditions over a $k$ range of 3.5-18.0 $\AA^{-1}$. The listed coordination numbers $\left(N_{\text {cor }}\right)$ have been corrected for the difference in distance between the reference and the analysis results. Data: synchrotron where the data were measured. Ref: synchrotrons where the reference was measured.

\begin{tabular}{llcccccc}
\hline \hline Foil & \multicolumn{1}{c}{ Data } & Ref & $N_{\text {cor }}$ & $R(\AA)$ & $\Delta \sigma^{2}\left(\times 10^{-5} \AA^{2}\right)$ & $\Delta E_{0}(\mathrm{eV})$ & $k^{1}$ variance \\
\hline Cu & SRS 8.1 & SRS 9.2 & 12.37 & 2.565 & 91 & -0.86 & 0.6 \\
Rh & SSRL 1.5 & SRS 9.2 & 13.59 & 2.684 & -69 & 0.65 & 0.8 \\
Pt & SRS 9.2 & SSRL 1.5 & 11.89 & 2.769 & 16 & 0.74 & 0.1 \\
\hline \hline
\end{tabular}

end of the interval were varied separately. To emphasize the low- or high- $k$ region in the analysis the $k$ weighting in the analysis was varied. Comparisons with different starting points to the analysis interval were done with $k^{1}$ weighting and comparisons with different end points to the analysis interval were carried out with $k^{3}$ weighting. The agreement between the Fourier filtered and unfiltered spectrum increases when the range of comparison is shortened. The decrease in variance is larger at the start than at the end of the analysis range. Hence truncation errors are larger at lower $k$ than at higher $k$. Shortening by 1.0 $\AA^{-1}$ at the start and $0.8 \AA^{-1}$ at the end gives excellent agreement between original and Fourier filtered data $\left(k^{1}\right.$ variance 0.07). Using this range for fitting the Fourier filtered data leads to coordinations parameters which are nearly identical to the original values. Maximum devia-
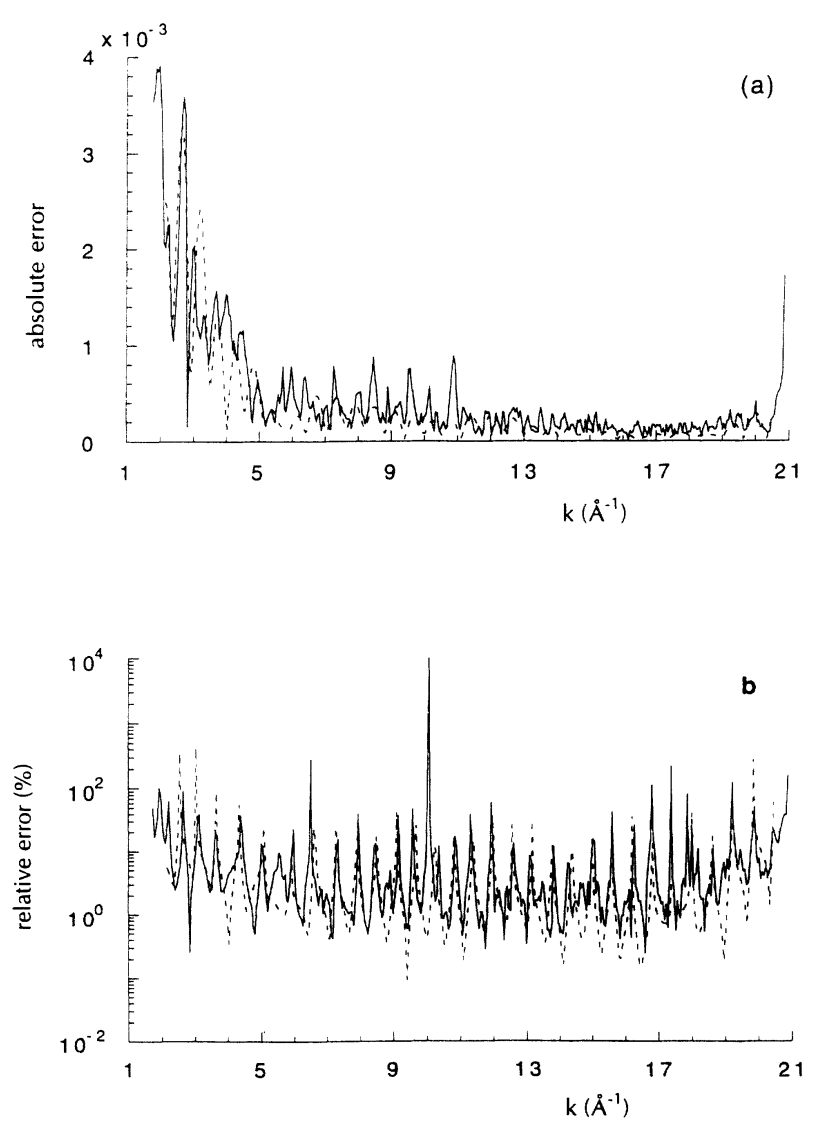

FIG. 2. Errors in the raw (solid line) and Fourier filtered (dashed line) EXAFS spectra of Pt foil measured at SRS 9.2. (a) Absolute errors and (b) relative errors. tions are coordination number $\pm 2 \%$, Debye-Waller factor $\pm 0.0002 \AA^{2}$, distance $\pm 0.003 \AA$, and $E_{0} \pm 0.5 \mathrm{eV}$.

While keeping the forward Fourier transform range at 2.3-19.4 $\AA^{-1}$, the end and start of the inverse Fourier transform range were varied separately. Either the end of the inverse Fourier transform range was fixed at $3.1 \AA$ or the start was fixed at $1.3 \AA$. The Fourier filtered spectra were analyzed over the interval $2.8-18.5 \AA^{-1}$ with the phase shift and backscattering amplitude used to cal-
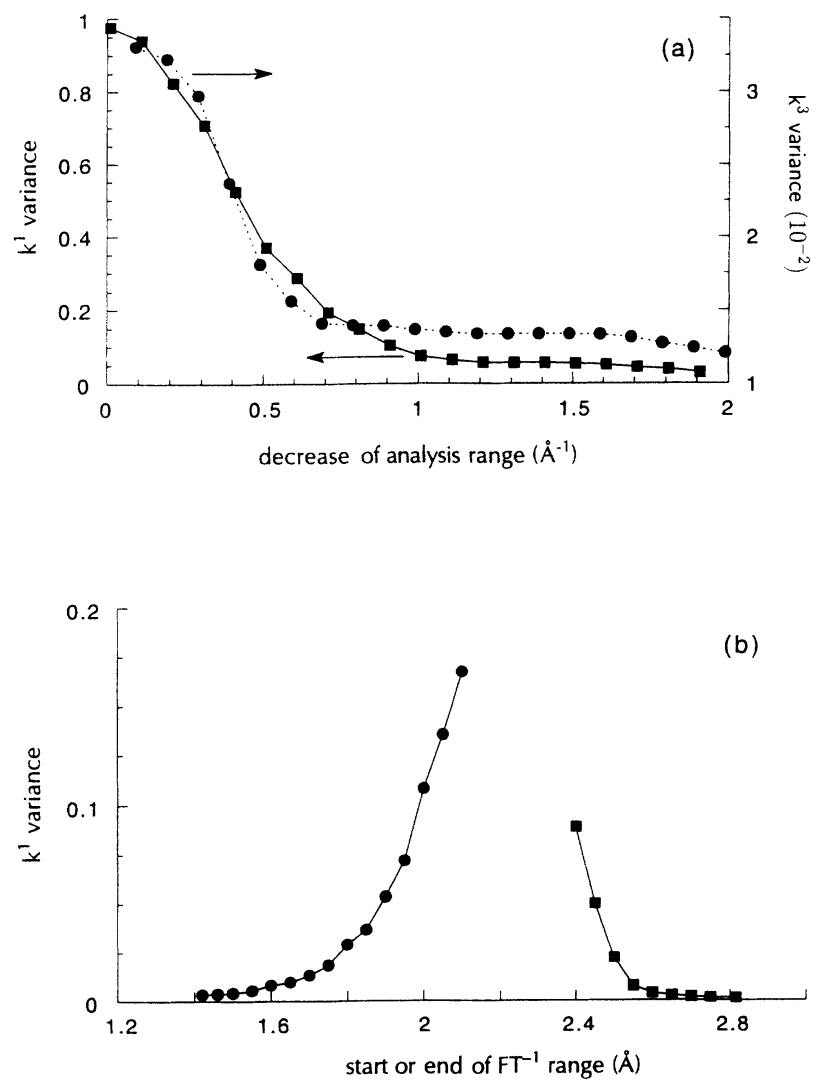

FIG. 3. Variance of the analysis of a Fourier filtered EXAFS spectrum with its original phase shift and backscattering amplitude. (a) Dependence of variance on the analysis range in $k$ space. The solid line with squares represents the increase of the start of the analysis range while keeping the end fixed at 18.5 $\AA^{-1}$. The dashed line with circles represents the decrease of the end of the analysis range while keeping the start fixed at 3.3 $\AA^{-1}$. (b) Dependence of variance for the $k^{1}$ weighted analysis from 3.5 to $18.0 \AA^{-1}$ on the inverse Fourier transform range. The circles represent the increase of the start of the inverse transform range. The squares represent the decrease of the end of the inverse transform range. 
culate the trial first-shell EXAFS spectrum. Shortening the range of the inverse Fourier transform results in an increase in the $k^{1}$ variance from 0.07 to 20 [Fig. 3(b)]. As long as the complete main peak is included in the inverse Fourier transform, excellent agreement over the complete analysis range can be obtained. Omitting only a small part of the main peak results in relatively large errors over the complete Fourier transform range. Changing the start and/or end of the forward Fourier transform range from nodes to maxima and minima does not result in a significant increase in the variance.

\section{Errors induced by changes in experimental conditions}

Changes in experimental conditions (temperature, higher harmonic rejection, etc.) affect the measured EXAFS spectrum. To estimate the magnitude of these changes we compared spectra measured under different experimental conditions. The comparison was quantified by analyzing the first shell of an EXAFS spectrum measured at one synchrotron with phase shift and backscattering amplitude functions extracted from the EXAFS spectrum of the same foil measured under different experimental conditions. The results of this analysis are listed in Table II. There is excellent agreement between the experimental spectra as shown by the very low variance values. The distances are within 0.005 $\AA$ of the expected values and both the Debye-Waller factor and the inner-potential shift are very close to the expected value of 0 .

The coordination numbers derived for copper and platinum foil deviate by less than $0.4(4 \%)$ from the expected value of 12 . The coordination number of 13.6 found in analyzing rhodium foil data measured at SSRL with a reference measured at SRS deviates by $13 \%$ from the expected value of 12 . The channel-cut monochromators used in these measurements do not allow any higher harmonics rejection and therefore this result indicates the
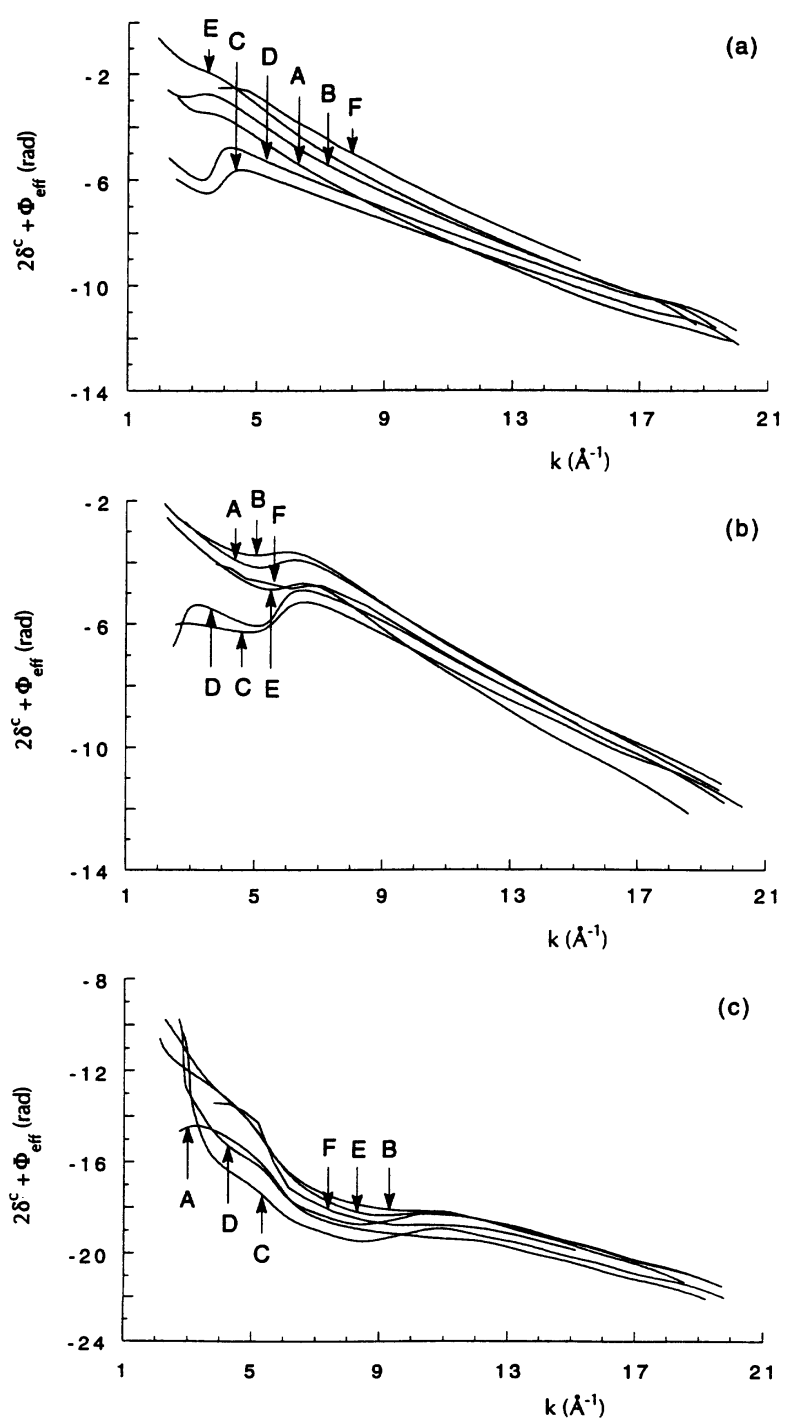
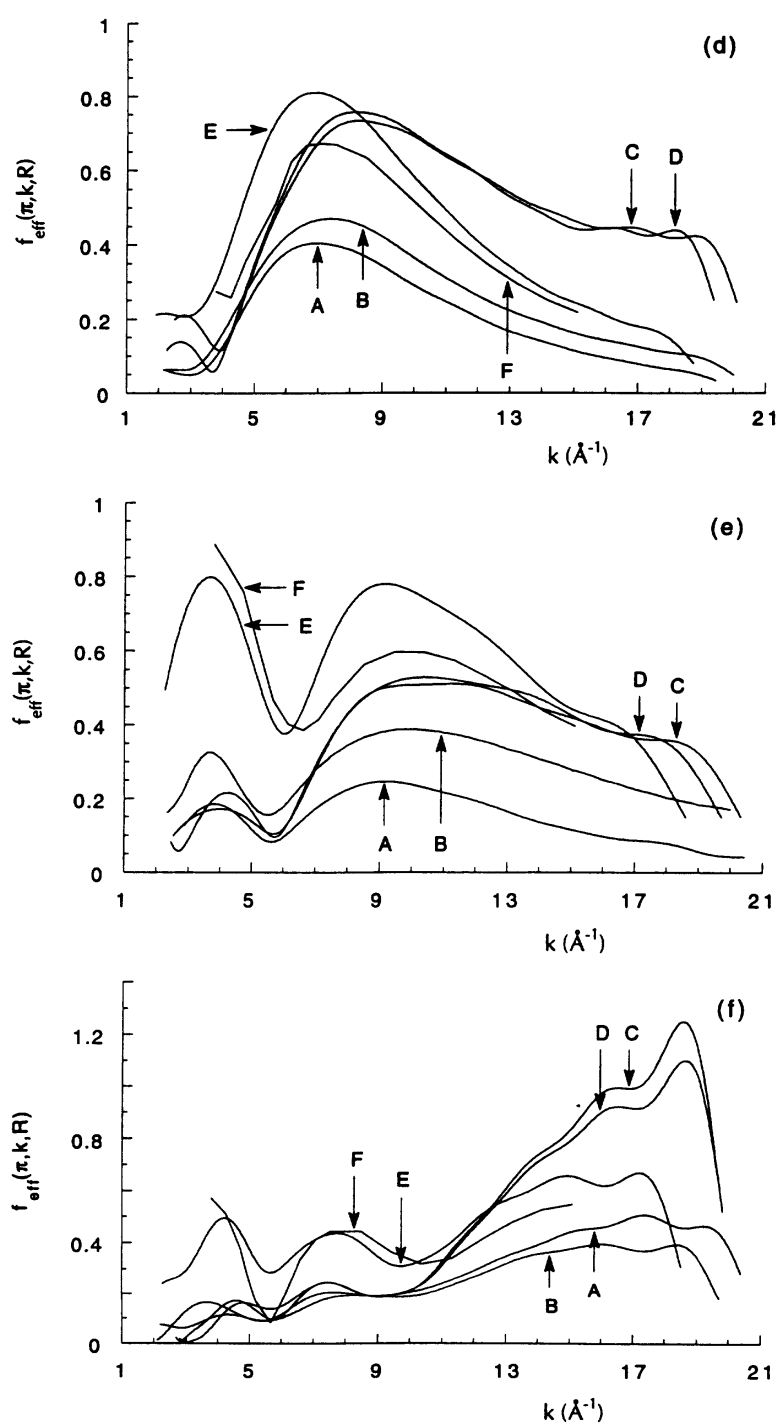

FIG. 4. Phases and backscattering amplitudes. $A$, experiment measured at SRS 9.2; $B$, FEFF; $C$, EXCURV90; $D$, MUFPOT; $E$, McKale et al., F, Teo and Lee. (a) Phase of copper, (b) phase of rhodium, (c) phase of platinum, (d) backscattering amplitude of copper, (e) backscattering amplitude of rhodium, and $(f)$ backscattering amplitude of platinum. 
magnitude of the systematic error that can be introduced by higher harmonics. The critical energy of the SSRL is lower than the critical energy of the Wiggler beamline at SRS. Hence the data measured at the SRS contain more higher harmonics. This will result in too low a backscattering amplitude in the reference and consequently a high apparent coordination number for unknowns analyzed with this backscattering amplitude. The platinum foil data are not affected because detection of higher harmonics was decreased by adjusting the gas fillings of the ion chambers

\section{Phase shifts and backscattering amplitudes}

Phase shifts and backscattering amplitudes were extracted from experimental data and FEFF, EXCURV90, and MUFPOT by Fourier filtering; filtering ranges are listed in Table III. The experimental phase shifts and backscattering amplitudes were extracted from the spectra measured at SRS 9.2 for copper and platinum and from the spectrum measured at SSRL 1.5 for rhodium. All theoretical phase shifts and backscattering amplitudes were calculated using the parameters listed in Table I. Phase shifts and backscattering amplitudes as tabulated by Teo and Lee and McKale et al. were generated directly from the tables to maximize the range usable for data analysis.

Backscattering amplitudes and phase shifts from the experimental data are shown in Fig. 4 together with their calculated values. The phase shifts from experimental data are parallel to the phase shifts calculated with the Hedin-Lundqvist exchange potential and also to the phase shifts derived from the tables of Teo and Lee or the tables of McKale et al. The shift has a maximum value of $2 \mathrm{rad}$. The phase shifts calculated with the $X \alpha$ exchange potential deviate from the experimental values as a function of electron energy. At high energy the agreement is quite good, but for $k$ values smaller than $5 \AA^{-1}$ deviations become as large as $\pi$. The backscattering amplitudes have the same shape, showing one maximum for copper, two for rhodium, and three for platinum. However, the relative amplitude of the maxima is not the same for the experimental data and the theoretical standards. Amplitudes that are too high at high- $k$ values will result in inflated Debye-Waller factors. If the relative amplitude of the maxima agrees with the experimental data only a scaling factor is needed.

\section{E. Comparison of theoretical methods}

The comparisons were carried out with $k^{1}$ weighted EXAFS spectra to emphasize the differences at low $k$. Our choice of $k^{1}$ weighted comparisons was based on the

TABLE III. Data ranges for Fourier filtering.

\begin{tabular}{lcc}
\hline \hline \multicolumn{1}{c}{ Data set } & $\begin{array}{c}\text { Forward FT range } \\
\left(\AA^{-1}\right)\end{array}$ & $\begin{array}{c}\text { Inverse FT range } \\
\left(\AA^{-1}\right)\end{array}$ \\
\hline Copper foil & $2.2-19.4$ & $1.0-3.0$ \\
Rhodium foil & $2.9-24.4$ & $1.2-3.0$ \\
Platinum foil & $2.2-20.3$ & $1.1-3.1$ \\
\hline \hline
\end{tabular}

importance of the low- $k$ region in EXAFS data analysis. Virtually all applications of EXAFS consist of measuring the XAFS of relatively heavy atoms in a matrix of low- $Z$ atoms. To determine the contribution of these low- $Z$ scatterers, analysis of the heavy scatterers in the low- $k$ part of the spectrum has to be accurate. Errors in phase or backscattering amplitude of heavy scatterers at low- $k$ will perturb the calculated EXAFS signal at low- $k$ and thus results in unreliable results for low- $Z$ scatterers. We also carried out $k^{3}$ weighted comparisons. Differences shown by such comparisons are much smaller and the parameters obtained more accurate.

To obtain the experimental first-shell $\chi$ functions that have been analyzed, the spectra plotted in Fig. 1 were Fourier filtered. Table III gives the data ranges for the forward and the inverse Fourier transforms. To minimize the influence of Fourier filtering truncation errors on the results of the data analysis the interval for data analysis was set to $3.50-18.00 \AA^{-1}$. This is equal to the
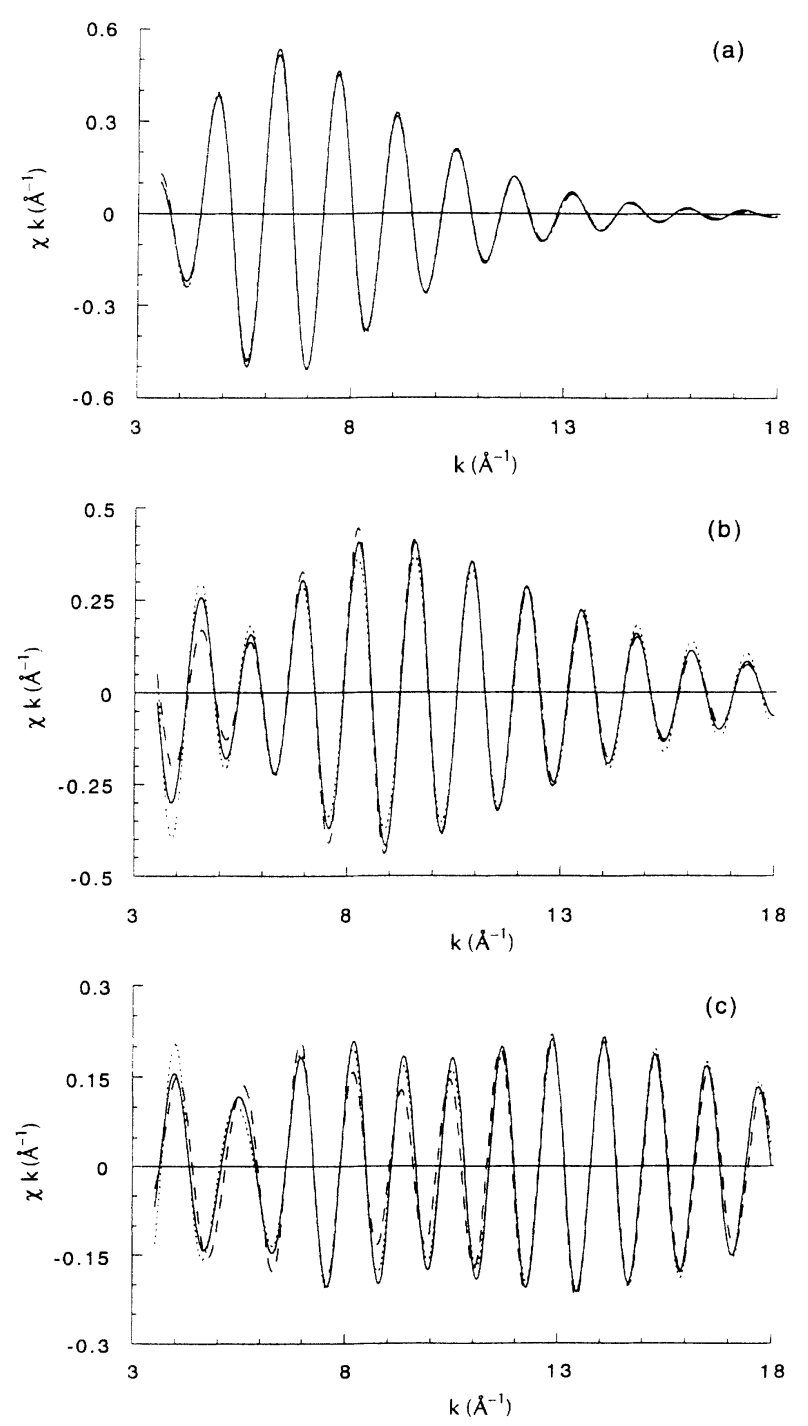

FIG. 5. Fit of experimental data (solid line) and a model function calculated with phase and backscattering amplitude for FEFF (dotted line) or EXCURV90 (dashed line) for (a) copper, (b) rhodium, and (c) platinum. 
TABLE IV. Numerical results of the $k^{1}$ weighted analysis of copper foil over a $k$ range of 3.5-18.0 $\AA^{-1}$. The listed coordination numbers $\left(N_{\text {cor }}\right)$ have been corrected for the difference in distance between the reference and the analysis results.

\begin{tabular}{lccccc}
\hline \multicolumn{1}{c}{ Standard } & $N_{\text {cor }}$ & $R(\AA)$ & $\Delta \sigma^{2}\left(\times 10^{-5} \AA^{2}\right)$ & $\Delta E_{0}(\mathrm{eV})$ & $k^{1}$ variance \\
\hline FEFF & 11.01 & 2.542 & 166 & -3.63 & 1.2 \\
EXCURV90 & 10.27 & 2.534 & 395 & 13.90 & 2.3 \\
MUFPOT & 10.00 & 2.527 & 416 & 9.27 & 2.6 \\
McKale et al. & 5.57 & 2.547 & 68 & -9.38 & 6.2 \\
Teo and Lee & 7.73 & 2.499 & 220 & -7.37 & 40.7 \\
\hline \hline
\end{tabular}

forward Fourier transform range plus at least $0.6 \AA^{-1}$ at the start and minus at least $0.8 \AA^{-1}$ at the end. The phase shifts and backscattering amplitudes plotted in Fig. 4 were used in the analysis. The structural parameters $N$, $R, \Delta \sigma^{2}$, and $\Delta E_{0}$ were optimized by nonlinear leastsquares fitting in $k$ space.

In Fig. 5 the experimental data are compared to the EXAFS spectra calculated with the $X \alpha$ and the HedinLundqvist exchange correlation potential over the complete data range for comparison. Spectra calculated with other theoretical standards have been omitted for clarity. Figure 6 shows the differences between the experimental data and the fits obtained for the different theoretical standards over a shorter data range. Numerical results are listed in Tables IV-VI for copper, rhodium, and platinum foils, respectively.

The copper foil spectra in Figs. 5(a) and 6(a) show that above $4.5 \AA^{-1}$ hardly any differences between the spectra are detectable. Below $4.5 \AA^{-1}$ differences in amplitude of up to $15 \%$ are evident. The parameters listed in Table IV show that theoretical references refine to a distance of 0.02-0.04 $\AA$ too short. The tables of Teo and Lee refine to a distance $0.06 \AA$ too short. Coordination numbers are as expected from the plots of the backscattering amplitudes with the highest backscattering amplitude giving the lowest coordination number. Variance values in Table IV show that a reference calculated with a HedinLundqvist exchange potential (FEFF) yields the best fit. EXCURV 90 and MUFPOT are nearly identical in fit quality. Fit quality achieved with the tables of Teo and Lee is poor.

Results for rhodium foil are plotted in Figs. 5(b) and 6(b) and listed in Table V. By comparing Figs. 5(a) and 5 (b) it is evident that the agreement between the different spectra is lower for rhodium than for copper. Only above $10 \AA^{-1}$ are the differences very small. Below $10 \AA^{-1}$ the differences in amplitude increase with decreasing $k$ value. Furthermore below $7 \AA^{-1}$ differences in phase are detected as evidenced by shifted nodes. The derived distances listed in Table $\mathrm{V}$ deviate less than $0.02 \AA$ from the expected value with the exception of the value determined from the McKale et al. tables. This deviates by almost $0.04 \AA$. The derived coordination numbers are again as expected from the backscattering amplitude plots, but differences are larger than for copper foil. Variance values show that phase shift and backscattering amplitude functions calculated with a Hedin-Lundqvist (FEFF) or $X \alpha$ exchange potential (EXCURV) yield the best fit. Fit quality of MUFPOT and the tables of McKale et al. are nearly identical. Fit quality achieved with the tables of
Teo and Lee is somewhat lower.

Platinum foil results are shown in Figs. 5(c) and 6(c) and Table VI. Comparing Fig. 5(c) with Figs. 5(b) and 5(a) leads to the conclusion that the differences between the various references are larger for platinum than for copper or rhodium. Differences in both phase and ampli-
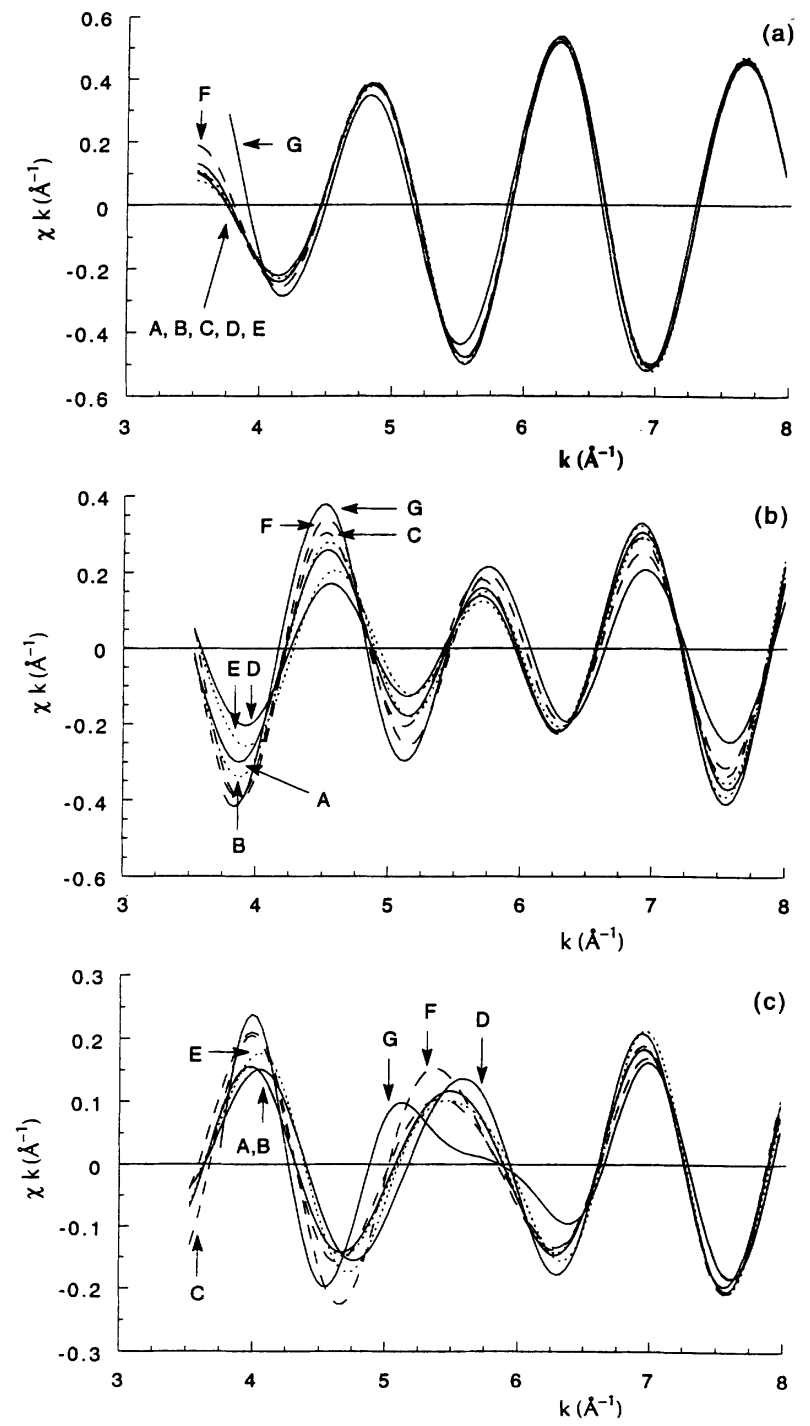

FIG. 6. Analysis results ( $\chi$ functions). $A$, experimental data; $B$, experimental reference; $C$, FEFF; $D$, EXCURV90; $E$, MUFPOT; $F$, McKale et al., G, Teo and Lee. (a) Copper data measured at SRS 8.1 and copper reference measured at SRS 9.2; (b) rhodium data measured at SSRL 1.5 and rhodium reference measured at SRS 9.2; (c) platinum data measured at SRS 9.2 and platinum reference measured at SSRL 1.5 . 
TABLE V. Numerical results of the $k^{1}$ weighted analysis of rhodium foil over a $k$ range of $3.5-18.0$ $\AA^{-1}$. The listed coordination numbers $\left(N_{\text {cor }}\right)$ have been corrected for the difference in distance between the reference and the analysis results.

\begin{tabular}{lrrrrr}
\hline \hline \multicolumn{1}{c}{ Standard } & $N_{\text {cor }}$ & $R(\AA)$ & $\Delta \sigma^{2}\left(\times 10^{-5} \AA^{2}\right)$ & $\Delta E_{0}(\mathrm{eV})$ & $k^{1}$ variance \\
\hline FEFF & 9.19 & 2.689 & 17 & -1.88 & 2.1 \\
EXCURV90 & 11.15 & 2.682 & 186 & 14.36 & 2.5 \\
MUFPOT & 10.57 & 2.671 & 184 & 12.36 & 3.2 \\
McKale et al. & 3.87 & 2.721 & -84 & 1.71 & 3.5 \\
Teo and Lee & 3.59 & 2.691 & -183 & 3.73 & 9.2 \\
\hline \hline
\end{tabular}

tude are evident below $12 \AA^{-1}$. Only reference spectra obtained from calculations with a Hedin-Lundqvist exchange potential give acceptable fits. The determined distances are, however, within $0.04 \AA$ of the expected value of $2.774 \AA$. Coordination numbers reflect the differences in backscattering amplitudes shown in Fig. 4(f). The tables of McKale et al., EXCURV90, and MUFPOT are nearly identical in fit quality. Fit quality achieved with the tables of Teo and Lee is again rather low.

Debye-Waller factors are heavily dependent on the type of phase shift and backscattering amplitude used. Calculations with $X \alpha$ exchange potentials always yield positive values. Calculations with a Hedin-Lundqvist exchange potential (FEFF), the tables of McKale et al., and the tables of Teo and Lee result in either positive or negative values with both tabulations giving large deviations in either direction.

It has been suggested that the accuracy of the backscattering amplitudes in the tables of McKale et al. and Teo and Lee can be improved by multiplying the backscattering amplitude with an energy-dependent mean free path term (4), which at high energies is equal to the constant complex self-energy used in EXCURV90 and MUFPOT,

$$
\exp \left(\frac{2 R V_{0}}{3.9 \sqrt{E}}\right) \text {. }
$$

We tested this for the tables of McKale et al. Analysis of the experimental data with these modified backscattering amplitudes and the original phase shifts resulted in an increase in coordination number $(14 \%)$ and $\Delta \sigma^{2}$ and a decrease in variance of $24 \%, 20 \%$, and $16 \%$ for copper, rhodium, and platinum foils, respectively.

The normalization procedure in the background subtraction influences the envelope of the derived $\chi$ function. Thus values of the Debye-Waller factor and the coordination number are subject to change. However, it has been shown ${ }^{5}$ that changing the normalization procedure from the step method to the "correct" McMaster normalization only affects the values of the Debye-Waller factors.

The values for $\Delta E_{0}$ are also dependent on the theoretical method used. Phase-shift and backscattering amplitude functions calculated with a Hedin-Lundqvist exchange potential yields negative values, the $X \alpha$ exchange potential gives rather large positive values, and the tables of McKale et al. and the tables of Teo and Lee show inconsistent behavior. The differences between the various codes are due to the different definition of the energy zero for the various theoretical standards. However, changes with element as in the tables of McKale et al. and the tables of Teo and Lee indicate an improper definition of the energy zero.

\section{F. Errors in derived parameters due to noise in the experimental data}

Due to the low noise level in the experimental data and the large interval used in the data analysis, errors in the derived parameters are very small. Maximum errors calculated from the data and the correlations between the parameters using standard procedures ${ }^{16}$ are as follows: coordination numbers 0.07 , distance $0.001 \AA, \Delta \sigma^{2}$ $\left(4 \times 10^{-5} \AA^{2}\right)$, and $\Delta E_{0}(0.6 \mathrm{eV})$.

\section{G. Statistical significance of differences in fit quality}

Although the variance values give an absolute indication of the agreement between experiment and theory, they are not a good criterion to evaluate whether these differences are real or whether they originate from the statistical errors in the experimental data. However, by applying an $F$ test $^{17}$ to the $\varepsilon_{v}^{2}$ (Ref. 6) values of the fits conclusions can be drawn about the significance of the differences between theoretical methods. Tables VII-IX

TABLE VI. Numerical results of the $k^{1}$ weighted analysis of platium foil over a $k$ range of $3.5-18.0$ $\AA^{-1}$. The listed coordination numbers $\left(N_{\text {cor }}\right)$ have been corrected for the difference in distance between the reference and the analysis results.

\begin{tabular}{lrrrrr}
\hline \multicolumn{1}{c}{ Standard } & $N_{\text {cor }}$ & $R(\AA)$ & $\Delta \sigma^{2}\left(\times 10^{-5} \AA^{2}\right)$ & $\Delta E_{0}(\mathrm{eV})$ & $k^{1}$ variance \\
\hline FEFF & 9.37 & 2.764 & -72 & -8.09 & 1.2 \\
EXCURV90 & 9.88 & 2.749 & 106 & 13.23 & 6.3 \\
MUFPOT & 10.17 & 2.739 & 130 & 6.00 & 6.1 \\
McKale et al. & 3.49 & 2.769 & -176 & -8.19 & 5.4 \\
Teo and Lee & 3.07 & 2.772 & -290 & -4.80 & 9.2 \\
\hline \hline
\end{tabular}


TABLE VII. Ratios (column/row) of $\varepsilon_{v}^{2}$ values ( $F$ values) of models calculated for first-shell $\mathrm{Cu}$ foil EXAFS. All models, except that of Teo and Lee, are calculated over a $k$ range of $3.5-18.0 \AA^{-1}$ and an $R$ range of 1.0-3.0 $\AA$. The model based on the tables of Teo and Lee is calculated over a $k$ range of $3.8-15.1 \AA^{-1}$. The 0.95 confidence limit of the $F$ distribution $\left[F\left(v_{\text {column }}, v_{\text {row }}, 0.95\right)\right]$ is 2.4 .

\begin{tabular}{lccccc}
\hline \multicolumn{1}{c}{ Standard } & FEFF & MUFPOT & EXCURV90 & McKale et al. & Teo and Lee \\
Experiment & 2.1 & 3.8 & 4.4 & 7.7 & 82.8 \\
FEFF & & 1.8 & 2.1 & 3.6 & 38.6 \\
MUFPOT & & & 1.2 & 2.0 & 21.8 \\
EXCURV90 & & & 1.7 & 18.7 \\
McKale et al. & & & & & 10.8 \\
\hline \hline
\end{tabular}

show ratios of the calculated $\varepsilon_{v}^{2}$ for all fits. Ratios between experimental and theoretical references are included for clarity. Comparison of these ratios with tabulated values of the $F$ distribution indicates that the difference in fit quality is statistically significant in a number of cases. From Table VII (copper foil) it is inferred that phase shift and backscattering amplitude functions calculated with a Hedin-Lundqvist exchange potential are in better agreement with experiment than functions calculated from the tables of McKale et al. or the tables of Teo and Lee. Although theoretical spectra calculated with the $X \alpha$ exchange potential yield higher variance values in the comparison with experiment than theoretical spectra calculated with a Hedin-Lundqvist exchange potential, the differences in variance are not large enough to infer that the spectra calculated with a Hedin-Lundqvist exchange potential are in better agreement with experiment than the spectra calculated with a $X \alpha$ exchange potential. The $\varepsilon_{v}^{2}$ ratios for rhodium foil (Table VIII) lead to the same conclusion: spectra calculated with either the $X \alpha$ or the Hedin-Lundqvist exchange potential are in better agreement with experiment than the spectra calculated with either the tables of McKale et al. or the tables of Teo and Lee. There is no statistically significant difference between the spectra calculated with either the $X \alpha$ or the Hedin-Lundqvist exchange potential. For platinum foil (Table IX) the spectrum calculated with the HedinLundqvist exchange potential is in better agreement with experiment than any of the other theoretical standards. There is no statistically significant difference between the spectra calculated with either the $X \alpha$ exchange potential or the tables of McKale et al. or the tables of Teo and Lee. The higher variance values for heavier elements indicate that theoretical calculation of EXAFS spectra of heavy elements is not as accurate as for light elements.
The ratios of the $\varepsilon_{v}^{2}$ values for the theoretical and experimental references indicate that experimental references are better than theoretical references. However, the limited availability of "perfect" experimental references means that in practice experimental standards may not necessarily be more accurate than theory. Furthermore, for complicated (biological) structures experimental references might not be available at all and therefore theoretical references have to be used.

\section{H. Physical reason of the differences between theoretical methods}

The physical reason why an energy-dependent selfenergy is required is because the exchange and correlation energies, which account for the interaction of the photoelectron with the other electrons in the material, are energy dependent. These two self-energy contributions correct the uncorrelated many-body wave function (the unpermuted product of one-particle wave functions) for the correlations introduced by the Pauli exclusion principle (the exchange energy) and the repulsion between the electrons due to their Coulomb interactions (the correlation energy). The Pauli exclusion principle makes the many-body wave function become zero when two electrons of the same spin occupy the same point, but does not affect the wave function if the electrons have opposite spin. Both correlations introduce a hole around the photoelectron consisting of a deficit of surrounding electrons. In the exchange case, the hole affects only those electrons with the same spin as the photoelectron, while in the correlation energy case both electron spins are affected equally. This hole lowers the interaction energy below what it would be if the wave function were

TABLE VIII. Ratios (column/row) of $\varepsilon_{v}^{2}$ values ( $F$ values) of models calculated for first-shell $\mathrm{Rh}$ foil EXAFS. All models, except that of Teo and Lee, are calculated over a $k$ range of $3.5-18.0 \AA^{-1}$ and an $R$ range of 1.2-3.0 $\AA$. The model based on the tables of Teo and Lee is calculated over a $k$ range of $3.8-15.1 \AA^{-1}$. The 0.95 confidence limit of the $F$ distribution $\left[F\left(v_{\text {column }}, v_{\text {row }}, 0.95\right)\right]$ is 2.5 .

\begin{tabular}{lccccc}
\hline \hline \multicolumn{1}{c}{ Standard } & EXCURV90 & FEFF & MUFPOT & McKale et al. & Teo and Lee \\
\hline Experiment & 2.3 & 2.6 & 3.2 & 5.4 & 13.2 \\
EXCURV90 & & 1.2 & 1.4 & 2.4 & 5.8 \\
FEFF & & & 1.2 & 2.0 & 5.0 \\
MUFPOT & & & 1.7 & 4.1 \\
McKale et al. & & & & & 2.4 \\
\hline \hline
\end{tabular}


TABLE IX. Ratios (column/row) of $\varepsilon_{v}^{2}$ values ( $F$ values) of models calculated for first-shell Pt foil EXAFS. All models, except that of Teo and Lee, are calculated over a $k$ range of $3.5-18.0 \AA^{-1}$ and an $R$ range of 1.0-3.0 $\AA$. The model based on the tables of Teo and Lee is calculated over a $k$ range of $3.8-15.1 \AA^{-1}$. Values in parentheses are the 0.95 confidence limit values of the $F$ distribution $\underline{\left[F\left(v_{\text {column }}, v_{\text {row }}, 0.95\right)\right]}$.

\begin{tabular}{lrrrrr}
\hline \multicolumn{1}{c}{ Standard } & FEFF & McKale et al. & Teo and Lee & \multicolumn{1}{c}{ MUFPOT } & EXCURV90 \\
\hline Experiment & $20.1(2.4)$ & $103.4(2.4)$ & $111.6(2.7)$ & $112.0(2.4)$ & $129.1(2.4)$ \\
FEEF & & $5.1(2.4)$ & $5.5(2.7)$ & $5.5(2.4)$ & $6.4(2.4)$ \\
McKale et al. & & & $1.1(2.7)$ & $1.1(2.4)$ & $1.2(2.4)$ \\
Teo and Lee & & & & $1.0(2.5)$ & $1.2(2.5)$ \\
MUFPOT & & & & & $1.2(2.4)$ \\
\hline \hline
\end{tabular}

uncorrelated because the Coulomb repulsion energy is decreased.

The size of each hole decreases with the increase of the energy of the photoelectron. In the exchange case the size of the hole is related inversely to the wave number of the photoelectron, while the Coulomb repulsion becomes less effective in keeping electrons apart as their relative energy increases. This decrease in the size of the correlation holes decreases the negative contribution of the exchange and correlation energies as the photoelectron energy increases and the Hedin-Lundqvist potential appears to approximate this energy dependence with sufficient accuracy for XAFS. FEFF contains the energy dependence of Hedin-Lundqvist potential and also includes the spherical wave features of the photoelectron. The tables of Teo and Lee include the Hedin-Lundqvist potentials also, but they make the plane-wave approximation to the photoelectron wave function, which is not as accurate as the spherical wave calculation. The other theories tested included spherical wave effects and used potentials accurate for low photoelectron energies, but did not allow them to change with energy. As we show in this paper, it is necessary to include both spherical wave effects and the energy dependence of the potential in order to obtain sufficient accuracy for the purposes of XAFS analysis.

\section{CONCLUSION}

The variance values found in the quantification of Fourier filtering errors (Fig. 3) is about an order of magnitude less than the errors found in the comparison of the theoretical standard. Coordination parameters are virtually unaffected by Fourier filtering. Fourier filtering errors can be minimized by using a smaller part of the filtered EXAFS spectrum than the original. Typically, the first $1.0 \AA^{-1}$ and the last $0.8 \AA^{-1}$ of the filtered EXAFS spectrum should not be used. Fourier filtering errors will have a very small influence on the structural parameters found in EXAFS data analysis.

Analysis of EXAFS spectra with theoretically obtained phase shifts and backscattering amplitudes will result in accurate distance determinations $( \pm 0.03 \AA)$. Other parameters vary with the type of calculation used. To obtain accurate coordination numbers a scaling factor, whose value will depend on the description of the exchange potential and the treatment of inelastic losses, is needed. The value of this scaling factor is not transferable from one element to another. Theoretically, it is expected that a scaling factor $\left(S_{0}^{2}\right)$ is required to correct the calculations for the decrease in the overlap of the passive electrons between the initial and final states of the absorbing atom. The required scaling factors to correct the coordination numbers are within the expected spread for the $a b$ initio calculations.

It appears that the choice of an energy-dependent selfenergy, as used in FEFF (Ref. 18) is the most important consideration and that the method of potential construction is secondary, at least in monoatomic metals. The use of ground state $X \alpha$ or energy-independent exchange, as in the McKale et al. tables or the codes EXCURV90 and MUFPOT, is found to be inadequate and leads to large phase and amplitude errors.

\section{ACKNOWLEDGMENTS}

We would like to thank the staff at SSRL, NSLS, and SRS for their assistance in the data collection. We thank Norman Binsted and Professor John Rehr for their stimulating interest in this research and their advice on how to operate EXCURV90, MUFPOT, and FEFF. The Dutch organization for Scientific Research provided travel funds to and beamtime at SSRL, NSLS, and SRS.

\footnotetext{
"Present address: Mitsubishi Kasei Corporation, Research Center, Chemical Laboratory, 1000 Kamoshida-cho, Midoriku, Yokohama 227, Japan.

†Present address: Debye Institute, University of Utrecht, P.O. Box 80083, 3508 TB Utrecht, The Netherlands.

${ }^{1}$ B. K. Teo and P. A. Lee, J. Am. Chem. Soc. 101, 2815 (1979).

${ }^{2}$ A. G. McKale, G. S. Knapp, and S.-K. Chan, Phys. Rev. B 33, 841 (1986).
}

${ }^{3}$ N. Binsted, S. L. Cook, J. Evans, G. N. Greaves, and R. J. Price, J. Am. Chem. Soc. 109, 3669 (1987).

${ }^{4}$ SERC Daresbury Laboratory MUFPOT program based on the work described in J. B. Pendry, Low Energy Electron Diffraction (Academic, London, 1974).

${ }^{5}$ J. Mustre de Leon, J. J. Rehr, S. I. Zabinsky, and R. C. Albers, Phys. Rev. B 44, 4146 (1991).

${ }^{6}$ F. W. Lytle, D. E. Sayers, and E. A. Stern, Physica B 158, 701 
(1988).

7J. J. Rehr, R. C. Albers, C. R. Natoli, and E. A. Stern, Phys. Rev. B 34, 4350 (1986).

${ }^{8}$ A. G. McKale, B. W. Veal, A. P. Paulikas, S.-K. Chan, and G. S. Knapp, J. Am. Chem. Soc. 110, 3763 (1988).

${ }^{9}$ D. C. Koningsberger and D. E. Sayers, Solid State Ionics 16, 23 (1985).

${ }^{10}$ J. W. Cook, Jr. and D. E. Sayers, J. Appl. Phys. 52, 5024 (1981).

${ }^{11}$ W. G. Wyckoff, Crystal Structures (Interscience, New York, 1974).

${ }^{12}$ E. A. Stern, B. A. Bunker, and S. M. Heald, Phys. Rev. B 21,
5521 (1980).

${ }^{13}$ L. J. Clarke, Surface Crystallography: An Introduction to Low Energy Diffraction (Wiley-Interscience, Chichester, 1985).

${ }^{14}$ G. Beni and P. M. Platzman, Phys. Rev. B 14, 1514 (1976).

${ }^{15}$ P. A. Lee and J. B. Pendry, Phys. Rev. B 11, 2795 (1975).

${ }^{16}$ W. H. Press, B. P. Flannery, S. A. Teukolsky, and W. T. Vetterling, Numerical Recipes. The Art of Scientific Computing (Cambridge University Press, Cambridge, 1989).

${ }^{17}$ A. J. Dobson, An Introduction to Generalized Linear Models (Chapman and Hall, London, 1990).

${ }^{18}$ EXCURV92 has an option to use an energy-dependent selfenergy. 